\section{JTI}

JOURNAL OF

TRAUMA AND INJURY

\title{
Successful Damage Control Resuscita- tion with Resuscitative Endovascular Balloon Occlusion of the Aorta in a Pediatric Patient
}

Received: June 3, 2020

Revised: July 14,2020

Accepted: July 17, 2020

\section{Correspondence to}

Dong Hun Kim, M.D.

Department of Trauma Surgery, Trauma Center, Dankook University Hospital, 201

Manghyang-ro, Dongnam-gu, Cheonan 31116 , Korea

Tel: +82-41-550-7119

Fax: +82-41-550-0039

E-mail:saint7331@gmail.com

\author{
Yoonjung Heo, M.D. ${ }^{1}$, Sung Wook Chang, M.D. ${ }^{2}$, Dong Hun Kim, M.D. ${ }^{1}$ \\ ${ }^{1}$ Department of Trauma Surgery, Trauma Center, Dankook University Hospital, Cheonan, \\ Korea \\ ${ }^{2}$ Department of Thoracic and Cardiovascular Surgery, Trauma Center, Dankook University \\ Hospital, Cheonan, Korea
}

Resuscitative endovascular balloon occlusion of the aorta (REBOA) is considered an emerging adjunct therapy for profound hemorrhagic shock, as it can maintain temporary stability until definitive repair of the injury. However, there is limited information about the use of this procedure in children. Herein, we report a case of REBOA in a pediatric patient with blunt trauma, wherein the preoperative deployment of REBOA played a pivotal role in damage control resuscitation. A 7-year-old male patient experienced cardiac arrest after a motor vehicle accident. After 30 minutes of cardiopulmonary resuscitation, spontaneous circulation was achieved. The patient was diagnosed with massive hemoperitoneum. REBOA was then performed under ongoing resuscitative measures. An intra-aortic balloon catheter was deployed above the supraceliac aorta, which helped achieved permissive hypotension while the patient was undergoing surgery. After successful bleeding control with small bowel resection for mesenteric avulsion, thorough radiologic evaluations revealed hypoxic brain injury. The patient died from deterioration of disseminated intravascular coagulation. Although the patient did not survive, a postoperative computed tomography scan revealed neither remaining intraperitoneal injury nor peripheral ischemia correlated with the insertion of a 7-Fr sheath. Hence, REBOA can be a successful bridge therapy, and this result may facilitate the further usage of REBOA to save pediatric patients with non-compressible torso hemorrhage.

Keywords: Shock, hemorrhagic; Balloon occlusion; Aorta; Child; Wounds and injuries 


\section{INTRODUCTION}

Hemorrhage is the most substantive contributor to potentially preventable mortality from trauma [1]. The interval between injury and definitive control of the bleeding focus is critical in reducing the incidence of preventable death. Resuscitative endovascular balloon occlusion of the aorta (REBOA) is an emerging adjunct therapy for profound hemorrhagic shock, as it can maintain temporary stability until definitive repair of the injury. Compared to resuscitative thoracotomy, REBOA is a less invasive procedure that allows earlier control of hemorrhage. According to the American Association for the Surgery of Trauma Aortic Occlusion for Resuscitation in Trauma and Acute Care Surgery registry, compared to resuscitative thoracotomy, REBOA has a survival benefit for patients with non-compressible truncal injury who undergo Zone 1 REBOA [2]. However, clinicians are still hesitant in implementing REBOA in children due to the immature anatomy of their vasculature, the unavailability of commercial catheters with small calibers, and insufficient data supporting its use. Herein, we report a case in which a 7-Fr REBOA catheter was deployed in a pediatric patient using a 7-Fr sheath via the common femoral artery, helping to achieve successful damage control resuscitation.

\section{CASE REPORT}

A 7-year-old male patient with no previous significant medical history experienced cardiac arrest after a motor vehicle accident, during which he was a backseat passenger. Initially, the patient was transported to a nearby hospital by an emergency medical service team. After 30 minutes of cardiopulmonary resuscitation, spontaneous circulation was achieved. He presented with profound hemorrhagic shock due to sustained blunt trauma in the left chest wall and the abdomen. The first responders performed endotracheal intubation and closed thoracostomy for left hemopneumothorax. An unfavorable outcome was expected due to suspected hypoxic brain damage caused by a long cardiac arrest time. However, the patient was still brought to our regional level 1 trauma center for the best possible treatment considering his age. The pre-hospital time from injury to arrival in the trauma center was 120 minutes.

Upon arrival, the patient was in extremis, with a blood pressure of 44/19 $\mathrm{mmHg}$ and minimal arterial pulsation. The Glasgow Coma Scale score was 3. According to the assessment of light reflex, the patient's pupils were fixed with a size of $6 \mathrm{~mm}$. The primary assessment revealed a seat belt sign and severe distention in the lower abdomen. Despite optimal resuscitation with crystalloid and blood
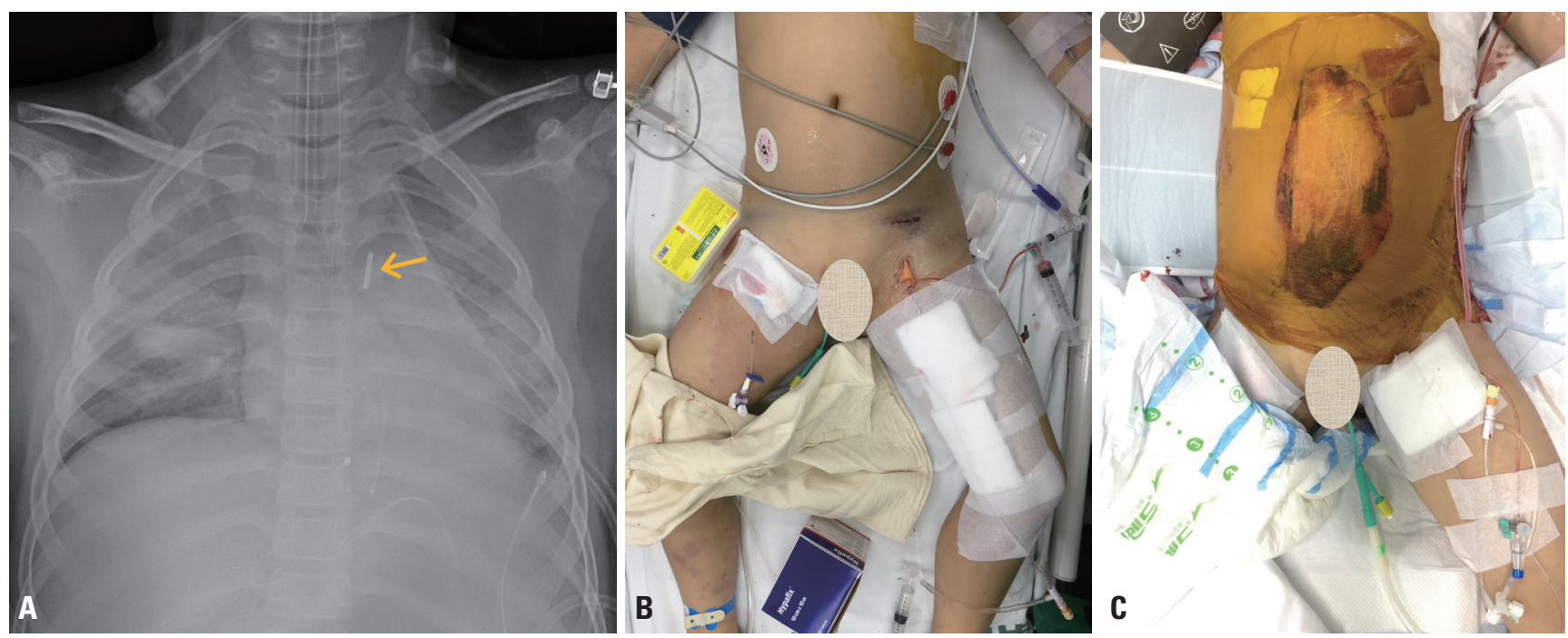

Fig. 1. (A) Position of the 7-Fr balloon catheter (arrow) in aortic Zone 1. (B, C) Preoperative and postoperative placement of the balloon catheter via the left femoral artery. 
transfusions, the patient did not have any hemodynamic response. The initial arterial blood gas analysis revealed a $\mathrm{pH}$ of 6.8 , lactate of $12.2 \mathrm{mmol} / \mathrm{L}$, and hemoglobin level of $3.8 \mathrm{~g} / \mathrm{dL}$. Only a small volume of blood drained through the indwelling chest tube. Thus, other major bleeding foci were suspected. Focused Assessment with Sonogra-

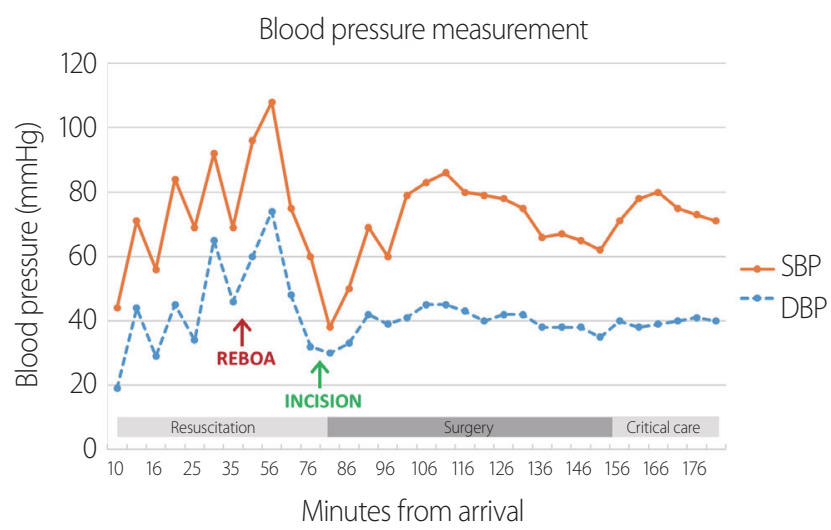

Fig. 2. Blood pressure measurements. The arrow indicates the time of REBOA inflation. REBOA: resuscitative endovascular balloon occlusion of the aorta, SBP: systolic blood pressure, DBP: diastolic blood pressure. phy for Trauma revealed massive intra-abdominal fluid collection. Thus, REBOA in Zone 1 was performed in the descending thoracic aorta under ongoing resuscitative measures [3]. A 7-Fr introducer sheath was placed in the left common femoral artery using the Seldinger technique. Then, a 7-Fr balloon catheter (RESCUE Balloon ${ }^{\mathrm{TM}}$, Tokai Medical Products, Aichi, Japan) was inserted along the guidewire. The balloon catheter tip was in the thoracic aorta as confirmed on serial radiography (Fig. 1A, B). The balloon was inflated with $5 \mathrm{~mL}$ of saline, which resulted in partial occlusion without inflated resistance in the syringe. The time from skin puncture to REBOA inflation was 8 minutes. The patient's hemodynamic status improved after balloon inflation (Fig. 2). Thus, he was wheeled into the operating room 61 minutes after the initial presentation.

Crash laparotomy revealed mesenteric avulsion with small bowel ischemia and a 5 -cm partial thickness laceration in the sigmoid colon. Numerous mesenteric arteries were lacerated. Considering these findings, we performed multiple small bowel resections without anastomosis and

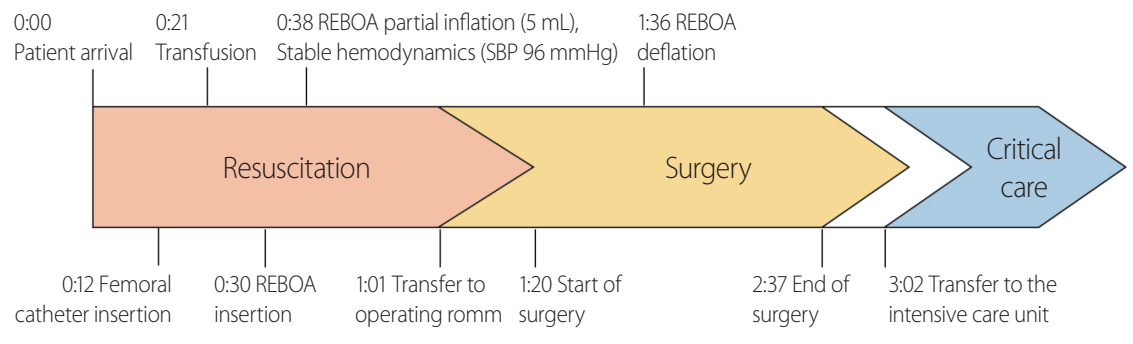

Fig. 3. Time course during REBOA performance (time interval from arrival). REBOA: resuscitative endovascular balloon occlusion of the aorta, SBP: systolic blood pressure.
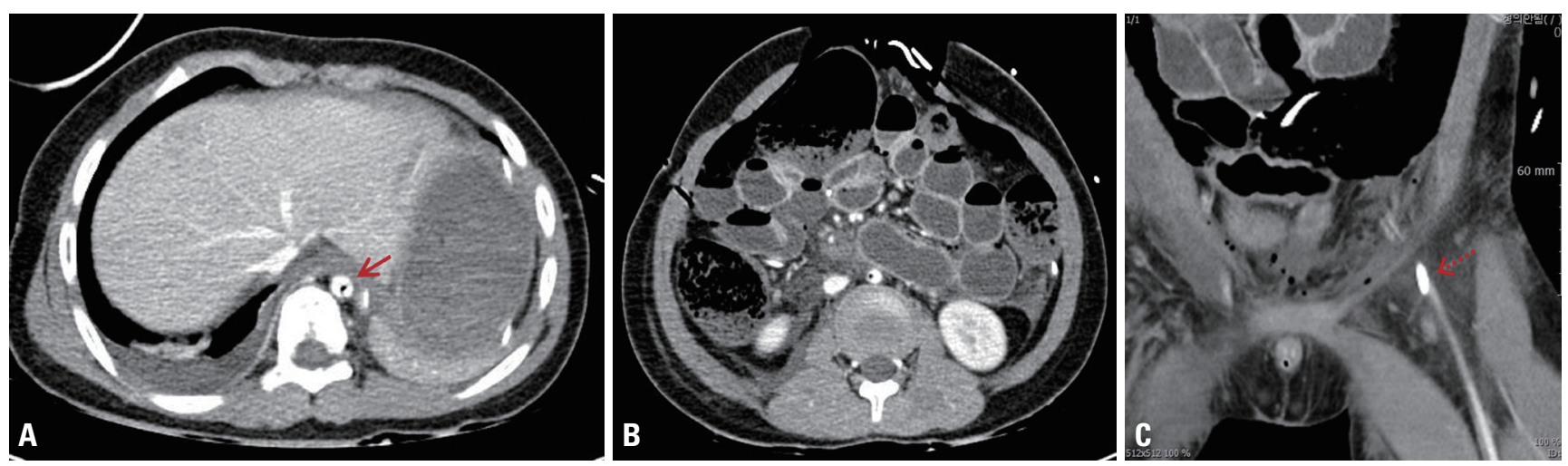

Fig. 4. Postoperative computed tomography scan of the abdomen. (A) A 7-Fr balloon catheter was accurately positioned in the aorta (arrow), and the aortic diameter was about $12.7 \mathrm{~mm}$. (B) There were no missed injuries or extravasation in the abdominal cavity. (C) A 7-Fr sheath placed in the left femoral artery (dotted arrow). Enhancement of the distal femoral artery indicated the absence of ischemic complications. 
primary repair of the sigmoid colon wall. To achieve a systolic blood pressure higher than $80 \mathrm{mmHg}$, the volume of the balloon, ranging from 8 to $3 \mathrm{~mL}$, was adjusted by a balloon holder. The balloon was completely deflated, with a total time of 58 minutes, immediately after the patient's vital signs stabilized during surgery. The operative time was 75 minutes, and the volume of blood loss during the surgery was approximately $1,500 \mathrm{~mL}$. The patient received three units of packed red blood cells (pRBC) and five units of fresh frozen plasma before REBOA insertion, as well as six units of pRBC during surgery. The catheter and the introducer sheath were left in the femoral artery for possible usage after the completion of damage control with temporary abdominal closure (Fig. 1C). The patient was transferred to the intensive care unit for ongoing resuscitation and organ support (Fig. 3).

Thorough radiologic evaluations were immediately performed for adjuvant diagnosis. Abdominopelvic computed tomography (CT) did not reveal any missed solid organ injury or active bleeding. In addition, the REBOA catheter was accurately positioned in Zone 1 , and there were no complications, including peripheral ischemia, attributed to REBOA placement (Fig. 4). However, severe hypoxic brain damage was noted on a brain CT scan. Unfortunately, the patient died secondary to multiorgan failure and deterioration of disseminated intravascular coagulation, possibly owing to the brain injury, approximately 19 hours after presentation.

\section{DISCUSSION}

The exact indications for REBOA placement are still debated. According to a joint statement from the American College of Surgeons Committee on Trauma and the American College of Emergency Physicians, the indications for REBOA are as follows [4]:

1. Patients arriving with traumatic life-threatening hemorrhage below the diaphragm, causing hemorrhagic shock, who are unresponsive or transiently responsive to resuscitation

2. Patients arriving in arrest from injury due to presumed life-threatening hemorrhage below the diaphragm

In our case, the patient fulfilled the indications for RE-
BOA by not responding to resuscitation and by presenting with massive hemoperitoneum caused by blunt trauma. However, there are no existing clinical guidelines or protocols regarding the patient's age, and whether these indications apply to pediatric patients has not been fully elucidated.

The major challenge in using REBOA in children is the selection of a proper introducer sheath and balloon catheter [5]. The aortic diameter and length in adults have been actively investigated for the utilization of REBOA. However, the relevance of these data for pediatric patients is unclear. The caliber of the common femoral artery grows with age and is associated with body mass and sex in adolescents [6]. However, the application of these findings to smaller children is limited. Furthermore, the inflation volume of REBOA should be appropriate to prevent vascular injuries, such as dissection, perforation, and rupture. In our case, the feasibility of placing a 7-Fr balloon catheter in a child with an aortic diameter of only $12.7 \mathrm{~mm}$ was validated (Fig. 3A). Carrillo et al. [7] showed the appropriate REBOA balloon inflation volumes for each Broselow category for Zones 1 and 3. Based on this study, to block aortic flow by $50 \%$, the optimal inflation volume in Zone 1 for a child who is $130 \mathrm{~cm}$ tall is $5.5 \mathrm{~mL}$. In our case, the maximal inflation volume was $8 \mathrm{~mL}$, which would be considered overinflation according to the previous study. The volume is also in accordance with the manufacturer's instructions, indicating that an inflation volume of $11 \mathrm{~mL}$ can establish a 20-mm balloon diameter (Table 1). However, the use of existing aortic occlusion balloons in children is arguable in terms of size. Thus, utmost caution is required when inflating the REBOA catheter in pediatric patients to prevent vascular complications. Unfortunately, no REBOA catheter for aortas with a diameter $<15$ $\mathrm{mm}$ is yet commercially available. There have been case reports of the off-label usage of Fogarty and 16-G Foley catheters to control vascular injuries in pediatric patients

Table 1. Balloon inflation parameters of the RESCUE balloon $^{\text {TM }}$ (Tokai RB-167080-E, Tokai Medical Products, Japan) (unpublished data)

\begin{tabular}{|lccccc|}
\hline Balloon diameter $(\mathrm{mm})$ & 20 & 25 & 30 & 35 & 40 \\
Inflation volume $(\mathrm{mL})$ & 11 & 15 & 21 & 28 & 40 \\
\hline
\end{tabular}


prior to the development of REBOA [8,9]. Although these catheters cannot be used as alternatives in actual clinical settings, the further accumulation of data could help in the development of pediatric endovascular balloons.

As there are several pediatric case reports worldwide, the use of REBOA in children may be widely accepted in the near future. An angioplasty balloon was deployed into the aorta via a 7-Fr sheath to improve the hemodynamic status of a 9-year-old girl, and this procedure eventually bought time for aortic endovascular stenting for aortoesophageal fistula repair [10]. A Japanese team inserted an infrarenal aortic occlusion balloon catheter via a $10-\mathrm{Fr}$ sheath for the primary repair of lacerated aortic bifurcation in a 12-year-old boy [11]. In our case, the patient did not survive. Nonetheless, REBOA can still be considered safe and feasible when a 7-Fr sheath is used, as shown in our patient, who is the youngest patient yet reported in whom REBOA was performed. However, more systematic high-quality studies must be conducted to assess the outcome and prognosis of REBOA in pediatric patients. Future studies should include morphometric analyses to completely validate the safety of the procedure. Developing a generalized protocol by building upon these novel experiences may open a new frontier in bridging therapy between injury and ultimate bleeding control in children.

\section{REFERENCES}

1. Eastridge BJ, Holcomb JB, Shackelford S. Outcomes of traumatic hemorrhagic shock and the epidemiology of preventable death from injury. Transfusion 2019;59:1423-8.

2. Brenner M, Inaba K, Aiolfi A, DuBose J, Fabian T, Bee T, et al. Resuscitative endovascular balloon occlusion of the aorta and resuscitative thoracotomy in select patients with hemorrhagic shock: early results from the American Association for the Surgery of Trauma's Aortic Occlusion in resuscitation for trauma and acute care surgery registry. J Am Coll Surg 2018;226:730-40.

3. Kim DH, Chang SW, Matsumoto J. The utilization of resuscitative endovascular balloon occlusion of the aorta: preparation, technique, and the implementation of a novel approach to stabilizing hemorrhage. J Thorac Dis 2018;10:5550-9.

4. Brenner M, Bulger EM, Perina DG, Henry S, Kang CS, Rotondo MF, et al. Joint statement from the American College of Surgeons Committee on Trauma (ACS COT) and the American College of Emergency Physicians (ACEP) regarding the clinical use of resuscitative endovascular balloon occlusion of the aorta (REBOA). Trauma Surg Acute Care Open 2018;3:e000154.

5. Campagna GA, Cunningham ME, Hernandez JA, Chau A, Vogel AM, Naik-Mathuria BJ. The utility and promise of resuscitative endovascular balloon occlusion of the aorta (REBOA) in the pediatric population: an evidence-based review. J Pediatr Surg 2020 Feb 1. doi: 10.1016/j.jpedsurg.2020.01.052 [Epub ahead of print].

6. Sandgren T, Sonesson B, Ahlgren R, Länne T. The diameter of the common femoral artery in healthy human: influence of sex, age, and body size. J Vasc Surg 1999;29:503-10.

7. Carrillo L, Skibber M, Kumar A, George M, Aziz S, T Harting $\mathrm{M}$, et al. Morphometric and physiologic modeling study for endovascular occlusion in pediatric trauma patients. ASAIO J 2020;66:97-104.

8. Gandolfo F, Filippelli S, Cetrano E, Carotti A. Managing major vessel injuries with a Fogarty catheter during chest re-opening in children. Interact Cardiovasc Thorac Surg 2013;17:216-7.

9. Black JJ, Allan A, Williams BT. The use of Foley balloon catheters in cardiac surgery. Eur J Cardiothorac Surg 1993;7:617-9.

10. Hill SJ, Zarroug AE, Ricketts RR, Veeraswamy R. Bedside placement of an aortic occlusion balloon to control a ruptured aorto-esophageal fistula in a small child. Ann Vasc Surg 2010;24:822.e7-9.

11. Kobayashi T, Matsuda K, Iwase F, Miyazaki Y, Amenomori S, Kikuchi $\mathrm{H}$, et al. Blunt abdominal aortic injury in a child: a case report. Nihon Kyukyu Igakukai Zasshi 2010;21:343-50. 